SECTION 31. Economic research, finance, innovation, risk management.

Anatoly Aleksandrovich Naumov

Docent, Candidate of Technical Sciences, corresponding member of International Academy of Theoretical \& Applied Sciences

Center of Applied Mathematical Research, Novosibirsk, Russia,

E-mail: a_a_naumov@mail.ru

\title{
ON THE CURRENT STATE OF ANALYTICAL METHODS OF STOCK MARKET VOLATILITY
}

\author{
Abstract: The paper discusses current approaches and models of stock markets index \\ volatility. \\ Keywords: Stock market, indexes, volatility, models.

\section{УДК 336.761: 336.748 \\ О СОВРЕМЕННОМ СОСТОЯНИИ МЕТОДОВ АНАЛИЗА ВОЛАТИЛЬНОСТИ ФОНДОВЫХ РЫНКОВ}

\begin{abstract}
Аннотация: В работе рассмотрень современнье подходы и модели решения задач анализа волатильностей индексов фондовых рынков.

Ключевые слова: Фондовый рынок, индексы, волатильность, модели.
\end{abstract}

Вопросы анализа волатильности фондовых рынков и их взаимного влияния друг на друга рассматриваются во многих работах. Цель настоящей заметки состоит в классификации результатов, полученных в этой области в последнее время, и в осмыслении этих результатов с теоретической и практической точек зрения. Практическая значимость методов решения таких задач очевидна. Если мы располагаем хорошим формальным аппаратом для прогнозирования поведения фондовых рынков, то в наших руках появляется инструмент по эффективному управлению финансовыми потоками, управлению фондовыми портфелями и т.д.

Математические модели измерения волатильности фондового рынка. В центре внимания многих работ (см. [1-3]) находится многомерная GARCH-модель под названием динамическая условная корреляция (Dynamic Conditional Correlation или DCC-GARCH). Модели GARCH - хорошо известные и распространенные инструменты для прогнозирования и анализа волатильности временных рядов, когда волатильность меняется с течением времени. Существует много многомерных моделей типа GARCH. Важной целью при построении многомерных моделей GARCH является то, чтобы сделать их достаточно простыми, но при этом сохранить их гибкость. DCC-GARCH модель является обобщением модели CCC-GARCH, когда корреляционная матрица зависит от времени. Модель DCC-GARCH имеет вычислительные преимущества в том, что число оцениваемых параметров не зависит от количества рядов, которые будут скоррелированы. Таким образом, могут быть оценены очень большие корреляционные матрицы.

Программное обеспечение задач анализа волатильности фондовых рынков. Использование специальных функций пакета программ $\mathrm{R}$ для решения задач анализа волатильности индексов фондового рынка приведено в работах [4; 5; 6]. В частности, в работе [6, с. 5] приведены описания функций: dcc.est (Dynamic conditional correlations), эта функция возвращает динамические условные корреляции; dcc.estimation (Estimating an DCC-GARCH model), эта функция осуществляет два этапа оценки DCC-GARCH 
модели и возвращает оценки, стандартизированные остатки, оцененные условные дисперсии и динамические условные корреляции.

Пример использования функции dcc.estimation [6]:

\# Simulating data from the original DCC-GARCH $(1,1)$ process

nobs <- 1000; cut <- 1000

a $<-\mathrm{c}(0.003,0.005,0.001)$

$\mathrm{A}<-\operatorname{diag}(\mathrm{c}(0.2,0.3,0.15))$

$\mathrm{B}<-\operatorname{diag}(\mathrm{c}(0.75,0.6,0.8))$

uncR <- matrix(c(1.0, 0.4, 0.3, 0.4, 1.0, 0.12, 0.3, 0.12, 1.0),3,3)

dcc.para $<-$ c $(0.01,0.98)$

dcc.data <- dcc.sim(nobs, a, A, B, uncR, dcc.para, model="diagonal")

\#\# Not run:

\# Estimating a DCC-GARCH $(1,1)$ model

dcc.results <- dcc.estimation(inia=a, ini $A=A$, iniB=B, ini.dcc=dcc.para,

dvar=dcc.data\$eps, model="diagonal")

\# Parameter estimates and their robust standard errors

dcc.results\$out

\#\# End(Not run)

Проблемы двумерных моделей. В некоторых работах (см., например, [11] и др.) рассмотрены двумерные модели оценивания волатильностей для пар индексов фондового рынка. Однако проблема использования таких моделей на практике заключается в том, что в волатильностях моделей, порожденных воздействием на индексы одновременно многих индексов, модели выделяют только вклад от двух из них. При этом вклад остальных индексов «размазывается» по оцененным параметрам модели, построенной для пары индексов. Выход достаточно простой, но требующий гораздо больших объемов вычислений, - использовать многомерные модели анализа волатильностей индексов фондового рынка $[1 ; 3 ; 7 ; 14 ; 15 ; 16]$.

Прогнозирование поведения фондовых рынков. Это одна из важнейших задач анализа поведения фондовых рынков. Ради решения этой задачи в основном и строятся модели волатильностей индексов. Именно решение такой задачи и представляется наиболее интересным исходя из практических потребностей. Схема решения таких задач проста: используя оцененную модель для волатильности, найти ее прогнозируемое значение [10; 15; 17].

Перспективные направления моделирования и решения задач оценивания волатильности. Предлагается построить модель для доходности, включающую в себя факторы внутренние и внешние для рынка (например, для индексов рынка включать в модель доходности отдельных инструментов данного рынка и индексы внешних рынков). Оценив параметры этой модели, найти для нее остатки. На основе остатков найти риски. Напомним, что волатильность в том виде, как она оценивается обычно с использованием дисперсий и стандартных отклонений, - это не самый удачный прием для оценивания рисков. Например, более интересным представляется модель рисков в виде VaR-оценок (квантилей). Кроме этого, предлагается использовать при анализе и прогнозировании доходностей инструментов пары «доходность-риск», как это делается, например, при анализе инвестиционных проектов. Можно, в частности, задачи управления финансовыми потоками и формирования портфелей рассматривать как задачи управления инвестиционным проектом с использованием соответствующего математического и программного обеспечения [19]. 


\section{References:}

1. Orskaug E. Multivariate DCC-GARCH Model - With Various Error Distributions. SAMBA/19/09, Juni 2009, 2009.

2. Almeida C., Vicente J. Are interest rate options important for the assessment of interest rate risk? - Working Papers Series from Central Bank of Brazil, No. 179, Research Department, 2009.

3. Silvennoinen A., Terasvirta T. Multivariate GARCH models. - Working Paper Series in Economics and Finance, SSE/EFI, No. 669, 2008.

4. Nakatani T., Terasvirta T. Testing for Volatility Interactions in the Constant Conditional Correlation GARCH Model. - Econometrics Journal, 2009, Vol. 12, pp. 147-163.

5. Nakatani T., Teraasvirta T. Appendix to Testing for Volatility Interactions in the Constant Conditional Correlation GARCH Model. - Department of Economic Statistics, Stockholm School of Economics, 2008. URL http://swopec.hhs.se/hastef/abs/hastef0649.htm (дата обращения: 23.04.14).

6. Nakatani T. Conditional Correlation GARCH models. Functions for estimating and simulating the family of the CC-GARCH models. Version 0.2.3. Date 2014-03-24, 2014.

7. Chib S., Omori Y., Asai M. Multivariate stochastic volatility// In: Handbook of Financial Time Series, Ed. by T.G. Andersen, R.A. Davis, J.-P. Kreiss, T. Mikosch. - New York: Springer, 2008.

8. Eklund B., Terasvirta T. Testing Constancy of the Error Covariance Matrix in Vector Models. - Journal of Econometrics, 2007, 140, pp. 753-780.

9. Engle R. Anticipating Correlations: A New Paradigm for Risk Management. - Princeton: Princeton University Press, 2009.

10. Skintzi V., Xanthopoulos-Sisinis S. Evaluation of Correlation Forecasting Models for Risk Management. - Journal of Forecasting, 2007, 26(7), pp. 497-526.

11. Kang S.H., Cho G.-H., S.-M. Yoon S.-M. Modeling sudden volatility changes: Evidence from Japanese and Korean stock markets. - Physica A, 2009, 388, pp. 3543-3550.

12. Kang S.H., Cheong G., Yoon S.-M. Structural changes and volatility transmission in crude oil markets. - Physica A, 2011, 390, pp. 4317-4324.

13. Kurmar D., Maheswaran S. Modelling asymmetry and persistence under the impact of sudden changes in the volatility of the Indian stock market. - IIMB Management Review 2012, 24, pp.123-136.

14. LaBarr A.D. Multivariate Robust Estimation of DCC-GARCH Volatility Model, A dissertation submitted to the Graduate Faculty of North Carolina State University in partial fulfillment of the requirements for the Degree of Doctor of Philosophy, Raleigh, North Carolina, 2010.

15. Bauwens L. Handbook of Volatility Models and Their Applications. - Wiley Handbooks in Financial Engineering and Econometrics, 2012.

16. Hafner C., Linton O. Efficient Estimation of a Multivariate Multiplicative Volatility Model. - Journal of Econometrics, 2010, 159(1), pp. 55-73.

17. Matei M. Perspectives on risk measurement: a critical assessment of PCGARCH against the main volatility forecasting models. - Romanian Journal of Economic Forecasting, 2012, 15(1), pp. 95-115.

18. Amado C., Terasvirta T. Modelling Volatility with Variance Decomposition. - Aarhus University, CREATES Research Paper 2011-1, 2011.

19. Наумов А.А. Методы анализа и синтеза инвестиционных проектов. Эффективность, риски, управление. - LAP LAMBERT Academic Publishing, 2013. 356 c. 